\title{
(6) OPEN ACCESS \\ Islet autoantibody status in a multi-ethnic UK clinic cohort of children presenting with diabetes
}

\author{
R Perchard ${ }^{1,2}$ D MacDonald, ${ }^{3}$ J Say, ${ }^{1}$ J Pitts, ${ }^{2}$ S Pye ${ }^{3}$ J Allgrove, ${ }^{1}$ \\ $\mathrm{K}$ Banerjee, ${ }^{2} \mathrm{R} \mathrm{Amin}^{4}$
}

${ }^{1}$ Department of Paediatrics, Royal London Hospital, London, UK

${ }^{2}$ Department of Paediatrics, Queens Hospital, Romford, UK ${ }^{3}$ Department of Immunology, Royal London Hospital, London, UK

${ }^{4}$ Clinical \& Molecular Genetics Unit, UCL Institute of Child Health, London, UK

Correspondence to Dr Rakesh Amin, Clinical Molecular and Genetis Unit, UCL Institute of Chlld Health, London

rakesh.amin@ucl.ac.uk

Received 20 April 2014 Revised 20 October 2014 Accepted 21 October 2014 Published Online First 19 November 2014
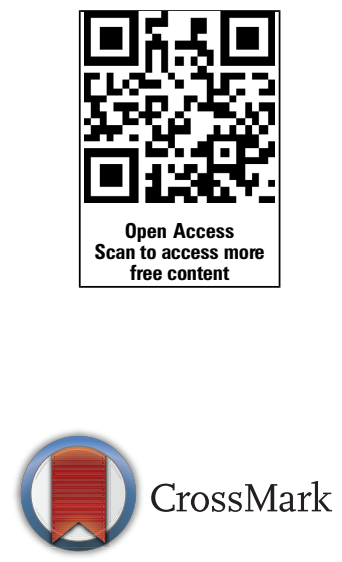

To cite: Perchard $R$, MacDonald D, Say J, et al. Arch Dis Child

2015;100:348-352.

\section{ABSTRACT}

Objective We prospectively determined islet autoantibody status in children presenting with diabetes to a single UK region in relation to ethnicity. Design 316 (68.0\% non-white) children presenting with diabetes between 2006 and 2013 were tested centrally for islet cell autoantibodies (ICA) and glutamic acid decarboxylase autoantibodies (GAD-65) at diagnosis, and if negative for both, tested for insulin autoantibodies (IAA). The assay used to measure GAD-65 autoantibodies changed from an in-house to a standardised ELISA method during the study.

Results Even with use of the standardised ELISA method, $25.8 \%$ of children assigned a diagnosis of type 1 diabetes still tested negative for all three autoantibodies. $30 \%$ of children assigned a diagnosis of type 2 diabetes were autoantibody positive, and these had the highest glycated haemoglobin ( $\mathrm{HbA} 1 \mathrm{c}$ ) levels at 12 months follow-up compared with other groups ( $p$ value for analysis of variance $<0.001$ ), although the sample size was small. Autoantibody positivity was similar between non-white and white children regardless of assay used $(60.0 \%(n=129)$ vs $56.4 \%(n=57)$ $\chi^{2}=0.9, p=0.35$ ), as was mean GAD-65 autoantibody levels, but fewer non-white children had two or more autoantibodies detectable $(13 \%(n=28)$ vs $27.7 \%$ $\left.(n=28), \chi^{2}=12.1, p=0.001\right)$.

Conclusions Islet autoantibody positivity was associated with a more severe phenotype, as demonstrated by poorer glycaemic control, regardless of assigned diabetes subtype. Positivity did not differ by ethnic group.

\section{INTRODUCTION}

The UK National Diabetes Audit shows that 9\% of adults have type 1A diabetes (T1D), with most of the remainder registered as having type 2 diabetes (T2D). ${ }^{1}$ For T1D compared with T2D adult populations, risk of vascular complications, intermediate outcomes and years of life lost are greater and treatment targets are less likely to be achieved. ${ }^{2}$ Given these adult outcomes, the possible effect of obesity on presentation with diabetes, ${ }^{3}$ and future prospects for interventions to preserve beta-cell function, ${ }^{4}$ it is increasingly important for paediatric diabetes practitioners to clearly define diabetes subtype for a child presenting with diabetes and align relevant care processes and treatments accordingly.

Islet autoantibodies are important markers of T1D-associated autoimmunity. ${ }^{4}$ However, the UK Prospective Diabetes Study (UKPDS) showed that $12 \%$ of adults with T2D in this trial tested positive for islet autoantibodies, and this was associated with increased future risk of requiring insulin. ${ }^{5}$ In a clinic

\section{What is already known on this topic}

Previous studies in this field, largely from the USA, indicate high prevalence of islet autoantibody positivity in children with a diagnosis of type 2 diabetes.

- The same studies demonstrate ethnic differences in islet autoantibody status.

\section{What this study adds}

- This is the first report from a contemporary UK paediatric diabetes clinic setting of islet autoantibody status in relation to clinical phenotype.

- Children and young people assigned a diagnosis of type 2 diabetes who are islet autoantibody positive have the poorest glycaemic control.

- In contrast with US studies, islet autoantibody positivity did not differ by ethnicity.

setting, the latest UK National Paediatric Diabetes Audit shows that $96.4 \%$ and $1.3 \%$ of children and young persons have been assigned a diagnosis of T1D and T2D, respectively. ${ }^{1}$ Yet, with more detailed investigation, the landmark US SEARCH study showed that $54.5 \%$ of young people had an autoimmune plus insulin-sensitive (IS) phenotype consistent with T1D, and $15.9 \%$ had a non-autoimmune insulin-resistant (IR) phenotype, consistent with $\mathrm{T} 2 \mathrm{D},{ }^{6}$ with the remainder having a mixed phenotype. In addition, there was evidence of ethnic differences in islet positivity during SEARCH: fewer non-white children (mostly Hispanic and Afro-American) had an autoimmune IS phenotype, and more had a non-autoimmune IR phenotype. ${ }^{6}$ In a separate US study, Libman $e t a l^{7}$ found that black children were less likely to have autoantibodies.

We determined islet autoantibody status in a clinic setting in children and young persons from a majority non-white (largely black African and South Asian) region of inner London who presented with diabetes between 2006 and 2013.

\section{METHODS}

\section{Patient selection}

Our cohort consisted of children from two paediatric diabetes centres (Queens Hospital in Romford 
and The Royal London Hospital) diagnosed with diabetes between January 2006 and April 2013 by specialist paediatric diabetes practitioners and aged $<19$ years. A diagnosis of T1D was assigned according to American Diabetes Association and International Society for Paediatric and Adolescent Diabetes guidelines after careful consideration by a paediatric diabetologist of the clinical presentation, biochemical abnormalities, the need for insulin and the total daily insulin dose, and the presence of islet autoantibodies. ${ }^{8} 9$ The diagnosis was reconsidered in light of symptoms and signs of a rarer form of diabetes (in which case genetic mutation analysis for monogenic diabetes was requested). If a child had negative islet autoantibody status, then a diagnosis of T1D was unaltered in the absence of other symptoms and signs-for example, insulin resistance or syndromic features. Diagnosis of T2D was considered in the presence of obesity, a family history of T2D and evidence of insulin resistance clinically (acanthosis nigricans), biochemically (normal or raised insulin and C-peptide levels during fasting or an oral glucose tolerance test and outside of the honeymoon period) and therapeutically (daily insulin requirements $>1.5$ units/kg and significant response to oral hypoglycaemic agents). Islet autoantibody status only became known at the following, usually the first, clinic appointment. Clinical and demographic data were prospectively collected during clinic visits and entered on to a dedicated diabetes database. Ethnicity was self-reported and coded according to the UK Office of National Statistics 2010 census guidelines. ${ }^{10}$ For this study, children and young persons were grouped into three categories: white, South Asian and black (migrants to the UK of black African descent). Ethics committee approval was not sought because no interventions were performed for the purpose of the study.

\section{Assays}

Blood samples for measurement of autoantibodies were collected by venepuncture at initial presentation, allowed to clot, and the serum separated as soon as possible to prevent haemolysis. Serum was stored at $2-8^{\circ} \mathrm{C}$. Blood samples were processed centrally at the immunology department at the Royal London Hospital and recorded electronically. The department operated a test selection policy whereby initially only islet cell antibodies (ICA) and glutamic acid decarboxylase (GAD-65) autoantibodies are measured, and, if these were negative, then insulin autoantibodies (IAA) were measured. A patient was considered autoantibody positive if at least one autoantibody was positive.

Before July 2007, samples were sent to the Protein Reference Unit at Sheffield, who used their in-house ELISA method for GAD-65 autoantibody measurement. ${ }^{11}$

\section{Indirect immunofluorescence: GAD-65 antibodies}

From August 2007 to December 2011, GAD-65 autoantibodies were measured using an indirect immunofluorescence technique. Samples were screened for GAD-65 antibodies using commercial monkey cerebellum slides ${ }^{12}$ (Instrumentation Laboratory Limited, UK) at a 1:10 dilution. The secondary antibody used was a monkey adsorbed fluorescein isothiocyanate (FITC)-conjugated polyclonal rabbit anti-human IgG (Instrumentation Laboratory Limited). Slides were viewed using a fluorescence microscope. Positive samples produced an apple-green fluorescence in the cytoplasm in the molecular layer, together with a less intense and patchy staining of the nerve terminals in the granular layer. Nuclei and glial cells are negative.

\section{ELISA: GAD-65 antibodies}

Since January 2012, sera were tested using a GAD-65 autoantibody (GAD-65) ELISA kit (RSR, UK). ${ }^{13}$ The wells of the microtitre plate were coated in a full-length human recombinant GAD-65. Any antibodies directed against any GAD-65 antigens present in the serum were bound to the microtitre plate. After a wash step, GAD biotin was added in a further incubation step, and a bridge formed between the GAD immobilised on the plate and the GAD biotin. The amount of GAD biotin bound is then determined by adding streptavidin peroxidase, which hydrolyses a chromogenic substrate $\left(3,3^{\prime}, 5,5^{\prime}\right.$-tetramethylbenzidine) to give a colour change. Acid was used to stop the reaction, and the intensity of colour was measured photometrically (450 and $405 \mathrm{~nm})$.

\section{Indirect immunofluorescence for ICA}

ICA were tested for using commercial monkey pancreas slides ${ }^{14}$ (Instrumentation Laboratory Limited) at a 1:2 dilution. The secondary antibody used was an FITC polyclonal rabbit antihuman IgG (Dako, UK). The slides were observed under a fluorescence microscope, and a sample was designated positive if the cytoplasm of all the islet cells was stained.

\section{Fluorescence enzyme immunoassay for IAA}

Samples that were negative for both ICA and GAD-65 autoantibodies were sent to the Protein Reference Unit at Sheffield for IAA testing, using fluorescence enzyme immunoassay on the Phadia250 (Thermo Scientific). It uses their ImmunoCAP Specific IgG methodology and is designed as a sandwich immunoassay. The insulin antigen, covalently coupled to the solid phase, reacts with any specific $\operatorname{IgG}$ antibodies present in the patient sample. After washing away of non-specific IgG antibodies, enzyme-labelled antibodies against $\operatorname{IgG}$ are added to form a complex. After incubation and a wash step, the bound complex is then incubated with a developing agent. After the reaction is stopped, the fluorescence of the eluate is measured. The higher the fluorescence, the more specific IgG antibodies are present in the sample. A cut-off of $5 \mathrm{mg} / \mathrm{L}$ was established following an internal evaluation and audit by the Protein Reference Unit.

\section{Statistical analysis}

Information on islet autoantibody status on the diabetes database was validated against results from the centralised immunology department electronic database at the Royal London Hospital. Given potential concerns that the assay method for measuring GAD-65 autoantibodies varied over three different time periods, for individuals assigned a diagnosis of T1D, we included data from any time point if they tested autoantibody positive. Those with T1D who tested autoantibody negative in the time periods up to January 2012 were excluded from analysis because of the limitations of the assays used to measure GAD-65 autoantibodies before this time - that is, for the analysis, T1D autoantibody-negative individuals were only from the time period after January 2012 as this is when the standardised GAD-65 autoantibody assay was used. Data on clinical and biochemical variables were summarised as means and compared between the four independent groups using a Student $t$ test or analysis of variance (ANOVA) model and by the $\chi^{2}$ test. For ANOVA testing, all variables were normally distributed, and we tested differences in variance between the four groups. SPSS V.16.1 was used for all analysis. A p value $<0.05$ was taken as significant.

\section{RESULTS}

Between January 2006 and April 2013, 316 children with diabetes had two or more islet autoantibody levels measured at 
Table 1 Islet autoantibody status during changes in GAD-65 autoantibody assay methods

\begin{tabular}{llllc}
\hline & Before July $\mathbf{2 0 0 7}$ & August 2007-December 2011 & After January 2012 & p Value \\
\hline Sample size (T1D/T2D) & $81(77 / 4)$ & $167(157 / 10)$ & $68(62 / 6)$ & Standardised ELISA \\
GAD-65 assay & In house ELISA & Indirect immunofluorescence & 66.7 & 0.61 \\
GAD-65 +ve (\%) & 18.4 & 13.8 & 45.0 & $<0.001$ \\
ICA +ve (\%) & 50.6 & 38.2 & 21.9 & 0.18 \\
IAA +ve (\%) & 26.5 & 24.0 & 72.2 & 0.88 \\
Positivity for any antibody (\%) & 64.6 & 49.1 & 50.0 & 0.002 \\
T2D with antibody positivity (\%) & 50.0 & 10.0 & 25.8 & 0.2 \\
T1D with antibody negativity (\%) & 32.4 & 44.2 & 0.09 \\
\hline
\end{tabular}

IAA, insulin autoantibody; ICA, islet cell autoantibody; GAD, glutamic acid decarboxylase; T1D, type 1A diabetes; T2D, type 2 diabetes.

diagnosis. Of these, 296 (93.7\%) were assigned a diagnosis of $\mathrm{T} 1 \mathrm{D}$, and the remainder were assigned a diagnosis of T2D. The latter group were all prescribed metformin therapy, and five were on once daily insulin glargine.

\section{Islet autoantibody status}

From 316 children presenting with diabetes during the study period, $192(60.8 \%)$ tested positive for any islet autoantibody using any assay. Median time from diagnosis of diabetes to islet autoantibody testing was 0.1 years (range 0-0.7). During the three time periods when the GAD-65 autoantibody assay changed from an in-house ELISA method (before July 2007) to an indirect immunofluorescence method (August 2007 to December 2011) and then a standardised RSR ELISA method (from January 2012), the frequency of GAD-65 positivity increased significantly and hence the frequency of children testing positive for any autoantibody increased (table 1). Mean GAD antibody levels for those who tested positive were 477.9 units/mL (SD 714.6).

We then compared the phenotype according to the assigned diabetes subtype and islet autoantibody status by dividing the cohort into four groups; T2D \pm autoantibody positivity and T1D \pm autoantibody positivity (the T1D autoantibody-negative group was restricted to those who tested negative after January 2012 as discussed in the methods, $n=68$ ).

Even with use of the standardised ELISA method for measurement of GAD-65 autoantibody, 25.8\% of children assigned a diagnosis of T1D still tested negative for all three autoantibodies (table 1), but no differences in phenotype were observed when compared with autoantibody-positive T1D children (table 2).
Children assigned a diagnosis of T2D were older at presentation and heavier than those with T1D. Some 30\% were autoantibody positive, and these had higher HbA1c at follow-up and body mass index (BMI) SD scores (SDSs) than the remainder of the cohort (table 2 and figure 1). Owing to the small sample, we were unable to evaluate any differences in insulin requirements.

There was no association with age and number of islet autoantibodies measured and the number of islet autoantibodies that tested positive. In addition, there was no association with age and islet autoantibodies positivity by ethnicity.

\section{Ethnicity}

Non-white children had a greater BMI SDS than white children (0.8 (1.9) vs 0.4 (1.4), p=0.001). In non-white (215 from 316 , $68.0 \%$ ) versus white children, ICA positivity was $38.1 \%$ vs $50.6 \%\left(\chi^{2}=3.4, p=0.06\right)$ and IAA positivity was $24.0 \%$ vs $25.5 \%\left(\chi^{2}=1.1, \mathrm{p}=0.66\right)$. GAD-65 positivity was $13.2 \%$ vs $36.4 \%\left(\chi^{2}=3.1, p=0.09\right)$ before July 2007 using the in-house ELISA method, $14.4 \%$ vs $12.1 \%\left(\chi^{2}=1.3, \mathrm{p}=0.49\right)$ between August 2007 and December 2011 using indirect immunofluorescence, and $67 \%$ vs $65 \%\left(\chi^{2}=0.7, p=0.84\right)$ after January 2012 using the standardised ELISA method. Mean GAD-65 antibody levels for those who tested positive for GAD-65 did not differ by ethnicity (non-white vs white; 345.1 (690.3) vs 309.5 (602.1) units/mL, $\mathrm{p}=0.84)$. Overall autoantibody positivity during the study period was similar between non-white and white children $\left(60.0 \% \quad(n=129)\right.$ vs $56.4 \% \quad(n=57), \chi^{2}=0.9$, $\mathrm{p}=0.35)$, but fewer non-white children had two or more autoantibodies detectable $\left(13 \%(n=28)\right.$ vs $27.7 \%(n=28), \chi^{2}=12.1$, $\mathrm{p}=0.001)$. HbA1c levels did not differ by autoantibody status in

Table 2 Phenotype in relation to diabetes subtype and islet autoantibody status

\begin{tabular}{|c|c|c|c|c|c|}
\hline & $\begin{array}{l}\text { T2D antibody +ve } \\
(n=6)(1.9 \%)\end{array}$ & $\begin{array}{l}\text { T1D antibody -ve } \\
(n=16)(25.8 \%)\end{array}$ & $\begin{array}{l}\text { T1D antibody +ve } \\
(n=186)(59.1 \%)\end{array}$ & $\begin{array}{l}\text { T2D antibody -ve } \\
(n=14)(4.4 \%)\end{array}$ & $\begin{array}{l}p \text { Value for } \\
\text { ANOVA }\end{array}$ \\
\hline Non-white, $\mathrm{n}(\%)$ & $4(66.7)$ & $12(75.0)$ & $139(74.7)$ & $11(79)$ & 0.45 \\
\hline Age at diagnosis of diabetes (years) & $12.5(3.7)$ & $10.6(4.6)$ & $9.1(4.3)$ & $14.1(1.7)$ & $<0.001$ \\
\hline Duration of diabetes (years) & $2.1(2.6)$ & $0.6(0.3)$ & $1.9(2.3)$ & $1.6(1.8)$ & 0.15 \\
\hline $\mathrm{HbA} 1 \mathrm{c}$ at diagnosis, \% (SD)mmol/mol & $\begin{array}{l}10.6(2.3) \\
92\end{array}$ & $\begin{array}{l}9.7(3.6) \\
83\end{array}$ & $\begin{array}{l}9.6(2.2) \\
81\end{array}$ & $\begin{array}{l}8.0(2.4) \\
64\end{array}$ & 0.03 \\
\hline $\begin{array}{l}\text { HbA1c } 12 \text { months after diagnosis, \% } \\
\text { (SD)mmol/mol }\end{array}$ & $\begin{array}{l}9.7(2.2) \\
8.3\end{array}$ & $\begin{array}{l}6.9(1.3) \\
52\end{array}$ & $\begin{array}{l}8.7(1.7) \\
72\end{array}$ & $\begin{array}{l}9.4(2.7) \\
79\end{array}$ & $<0.001$ \\
\hline BMI SDS at presentation & $2.0(2.2)$ & $0.6(1.4)$ & $0.7(1.6)$ & $1.5(1.7)$ & $<0.001$ \\
\hline On insulin treatment, $\mathrm{n}(\%)$ & $1(16.7)$ & $16(100)$ & $186(100)$ & $5(35.7)$ & 0.22 \\
\hline
\end{tabular}

The T1D autoantibody-negative group was restricted to those who tested negative after January 2012, as this is when the standardised RSR ELISA method for GAD-65 autoantibody measurement was used - that is, T1D-negative children diagnosed before January 2012 were excluded.

Unless otherwise noted, values are mean (SD).

BMI, body mass index; HbA1C, glycated haemoglobin; SDS, standard deviation score; T1D, type 1A diabetes; T2D, type 2 diabetes. 


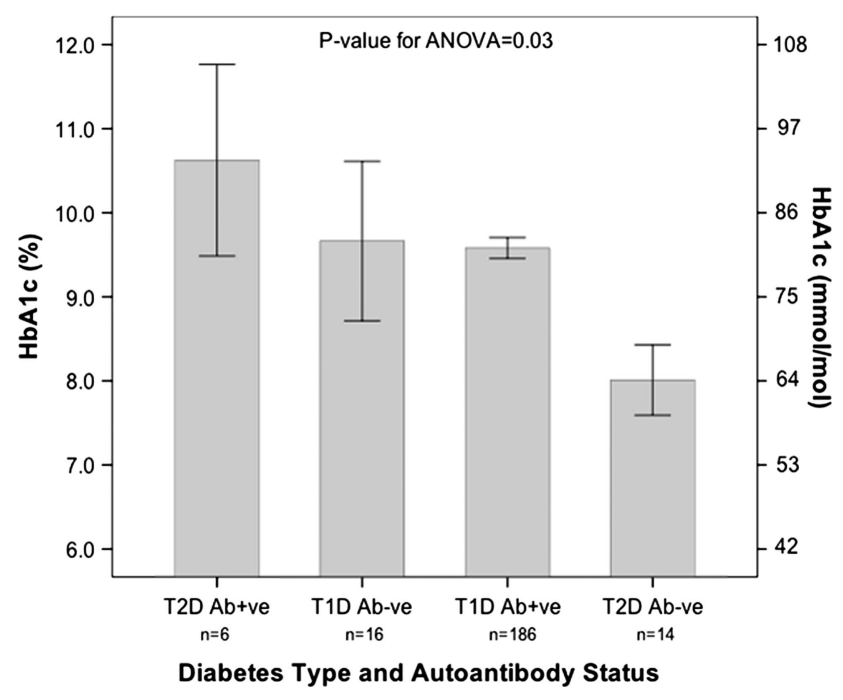

Figure 1 Glycated haemoglobin ( $\mathrm{HbA} 1 \mathrm{c})$ levels $( \pm \mathrm{SE})$ at diagnosis in relation to diabetes subtype and islet autoantibody status. The T1D autoantibody-negative group was restricted to those who tested negative after January 2012, as this is when the standardised RSR ELISA method for GAD-65 autoantibody measurement was used - that is, T1D-negative children diagnosed before January 2012 were excluded. Ab, antibody; ANOVA, analysis of variance; T1D, type 1A diabetes; T2D, type 2 diabetes.

the three ethnic groups. There were more girls in the non-white group $(n=121(62 \%)$ vs $n=48(40 \%), p<0.001)$, but no gender differences were found in autoantibody status. No differences were observed within ethnic groups (black African and South Asian).

\section{DISCUSSION}

In this multi-ethnic UK clinic cohort of childhood-onset diabetes, we aimed to determine the percentage of children assigned a diagnosis of T1D or T2D who tested positive for at least one autoantibody. Despite use of a standardised ELISA method for GAD-65 autoantibody, 25.8\% of children assigned a diagnosis of T1D still tested negative for all three autoantibodies, but no phenotypic difference existed in these compared with autoantibody-positive T1D children. Some 30\% of children assigned a diagnosis of T2D were autoantibody positive, and these had the highest HbA1c at the 12-month follow-up ( $\mathrm{p}$ value for ANOVA <0.001) and BMI ( $p$ value for ANOVA <0.001). We found no overall difference in rates of autoantibody positivity between ethnic groups at presentation with diabetes.

Studies that have added to our knowledge of the phenotype of children presenting with diabetes include the landmark US $\mathrm{SEARCH}^{6}$ and TODAY Studies, ${ }^{15}$ a study in German and Austrian youths, ${ }^{16}$ and the Childhood Diabetes in Finland Study. ${ }^{17}$ The TODAY and German/Austrian studies were limited to patients with T2D. Certain observations from these studies are relevant to our study. First, rate of islet autoantibody positivity appears to differ between studies-for example, during SEARCH, only 38\% of individuals were positive for both IAA and GAD-65 autoantibody, ${ }^{6}$ but during the Finnish Study, 91\% tested positive for both autoantibodies. ${ }^{18}$ Second, $15.1 \%$ of T1D children were autoantibody negative during SEARCH compared with $25.8 \%$ in our study, and this may reflect differences in assay standardisation, number of autoantibodies measured and population between studies. Other major islet autoantibody markers of T1D include zinc transporter 8 and IA2. ${ }^{18}$ Routine measurement of these autoantibodies in the immediate period after diagnosis increases the rate of autoantibody positivity. ${ }^{19}$ Third, differences exist in rates of autoantibody-positive T2D children between studies: $12.6 \%$, $9.8 \%$ and $15 \%$ of $\mathrm{T} 2 \mathrm{D}$ children were autoantibody positive during the SEARCH, TODAY and German/Austrian studies, respectively, compared with $30 \%$ in our UK clinic cohort. ${ }^{6} 1516$ This may reflect differences in criteria for diabetes classification, ethnicity and the other reasons mentioned above. We found that autoantibody-positive T2D children had the highest $\mathrm{HbA} 1 \mathrm{c}$ at the 12-month follow-up and BMI, suggesting a more severe phenotype. This is consistent with observations from SEARCH, where similar children had lower C-peptide levels and higher BMI, ${ }^{6}$ and from TODAY where similar children had the highest HbA1c level and lowest C-peptide. ${ }^{6}$ Finally, there was evidence of ethnic differences in autoantibody status in the SEARCH and TODAY studies. During SEARCH, more non-Hispanic white children were autoantibody positive compared with other ethnic groups. During TODAY, more non-Hispanic white T2D children were autoantibody positive, compared with other ethnic groups. ${ }^{15}$ In the German and Austrian study of youth with T2D, positivity was not compared between ethnicities. Our study found similar positivity between white, Asian and Black ethnic groups. These inconsistent outcomes require further investigation.

Although controversial, the blurred clinical distinction between T1D and T2D may be related to the increasing prevalence of obesity, ${ }^{20}$ but the impact this has on the natural history of diabetes is unclear. ${ }^{21}$ In adults with diabetes, it is unclear whether knowledge of islet autoantibody status changes management; evidence that positivity predicts insulin requirements in T2D in the UKPDS was based on a small sample size, and overall accuracy of the prediction was low and did not influence HbA1c response to medication $^{22}$ or rates of diabetic ketoacidosis. ${ }^{23}$ Similarly, in childhood epidemiological studies, islet positivity did not predict insulin requirements in patients with T2D. ${ }^{2425}$ However, other data indicate a link between islet autoantibody status and decline in betacell function, ${ }^{26}$ and, during euglycaemic clamps, children with islet autoantibody positivity and obesity have lower insulin levels ${ }^{27}$ and increased cardiovascular risk factors. ${ }^{28}$ Consistent with this, both the SEARCH and TODAY studies and, to a lesser extent, our study indicate a more severe phenotype in relation to autoimmune status. ${ }^{6}$ As yet, it is unclear whether early introduction of insulin therapy may be beneficial, and this requires further study.

\section{Limitations of the study}

Our sample size was small, and we did not have C-peptide measurements, HLA genotyping, measurements of insulin sensitivity or deep clinical phenotype to support some of our observations. It is likely that diagnostic accuracy may have changed over time; however, the same diagnostic criteria were applied to each patient throughout the study by the same physicians.

\section{Limitations of the assays}

The sensitivity and specificity for ICA indirect immunofluorescence are $85 \%$ and $98 \%$, and for GAD ELISA testing are $92 \%$ $(\mathrm{n}=50)$ and $98 \%(\mathrm{n}=100)$, respectively. The GAD ELISA has a cut-off of $\leq 5 \mathrm{U} / \mathrm{mL}$ and a lower detection rate at $+2 \mathrm{SDs}$ of $0.57 \mathrm{U} / \mathrm{mL}$. The interassay precision of the kit has been shown to have a coefficient of variation of $5.7 \%$ with a sample of $96.9 \mathrm{U} /$ $\mathrm{mL}$ and $5.2 \%$ for a sample of $21.0 \mathrm{U} / \mathrm{mL}(\mathrm{n}=20)$. The intra-assay precision was $7.3 \%$ for a sample of $97.2 \mathrm{U} / \mathrm{mL}$ and $8.5 \%$ for a sample of $20.0 \mathrm{U} / \mathrm{mL}(\mathrm{n}=25)$. Indirect immunofluorescence was the method used to test GAD-65 autoantibodies between August 2007 and December 2011. This method relies on a subjective assessment of the slides under a microscope, and is therefore subject to operator error. However, the results were always 
checked by a second operator, and, if there were any equivocal results, then the test was repeated. The laboratory also carried out monthly screening audits whereby known antibody-positive or -negative samples, including ICA and GAD autoantibodies, were tested and read by all screeners to ensure consistency in interpretation, and results were analysed, fed back and discussed. Another consideration is that the filters and optics of the microscope will influence the sensitivity of the kit, ${ }^{12}$ but this was minimised by regular testing and maintenance of the microscope. A recognised limitation of ICA testing with immunofluorescence is the potential for another autoantibody, such as anti-nuclear antibody or antimitochondrial antibody, to hide or be confused with GAD-65. This could potentially also be relevant for GAD-65 autoantibody testing with immunofluorescence. ${ }^{14}$ These factors may have influenced our lower sensitivity for GAD-65 autoantibodies using indirect immunofluorescence compared with ELISA. All test methods would be run with internal quality controls and the testing laboratory would be Clinical Pathology Accredited accredited and participate in national external quality assurance (NEQAS), which highlights any issues with the laboratory processes or test protocols/methods. However, these controls and NEQAS samples would, in the majority of cases at least, be using adult sera and may not always be relevant in the paediatric population.

\section{Conclusions}

This is the first UK study to date that evaluates islet autoantibody status and phenotype in relation to specific ethnic groups in a cohort of both T1D and T2D children using information routinely collected during clinic appointments. The effect of islet autoantibody status on disease progression is unclear in those assigned a diagnosis of T2D and requires further study. Overall islet autoantibody positivity did not differ by ethnicity.

Contributors All authors listed meet the ICMJE recommendations for authorship. All authors listed made a substantial contribution to the design of the work or the acquisition, analysis or interpretation of data for the work. RP, DMacD and RA drafted the work, and all authors revised it critically for important intellectual content.

\section{Competing interests None.}

Ethics approval Not required.

Provenance and peer review Not commissioned; externally peer reviewed.

Open Access This is an Open Access article distributed in accordance with the Creative Commons Attribution Non Commercial (CC BY-NC 4.0) license, which permits others to distribute, remix, adapt, build upon this work non-commercially, and license their derivative works on different terms, provided the original work is properly cited and the use is non-commercial. See: http://creativecommons.org/ licenses/by-nc/4.0/

\section{REFERENCES}

1 National Diabetes Audit 2010-2011. Report 1. Care Processes and Treatment Targets. http://www.selfmanagement.co.uk/sites/default/files/files/National_ Diabetes_Audit_2010_2011.pdf (accessed 27 Jul 2013).

2 National Diabetes Audit 2010-2011. Report 2: Complications and Mortality. http://www. hqip.org.uk/assets/NCAPOP-Library/NCAPOP-2012-13/Diabetes-Audit-Report-10-11ComplicationsMortality-pub-2012.pdf (accessed 27 Jul 2013).

3 Han JC, Lawlor DA, Kimm SY. Childhood obesity. Lancet 2010;375:1737-48.
4 Bluestone JA, Herold K, Eisenbarth G. Genetics, pathogenesis and clinical interventions in type 1 diabetes. Nature 2010;464:1293-300.

5 Turner R, Stratton I, Horton V, et al. UK Prospective Diabetes Study Group 1997 UKPDS 25: autoantibodies to islet-cell cytoplasm and glutamic acid decarboxylase for prediction of insulin requirement in type 2 diabetes. Lancet 1997;350:1288-93.

6 Dabelea D, Pihoker C, Talton JW, et al. Etiological approach to characterization of diabetes type. The SEARCH for Diabetes in Youth Study. Diabetes Care 2011;34:1628-163.

7 Libman IM, Pietropaolo M, Trucco M, et al. Islet cell autoimmunity in white and black children and adolescents with IDDM. Diabetes Care 1998; 21:1824-7.

8 American Diabetes Association. Position Statemement. Diagnosis and Classification of Diabetes Mellitus. Diabetes Care 2004;27:S5-10.

9 Craig ME, Hattersley A, Donaghue KC. (ISPAD Clinical Practice Consensus Guidelines 2009 Compendium). Definition, epidemiology and classification of diabetes in children and adolescents. Paediatr Diabetes 2009;10:3-12.

10 Office for National Statistics. Neighbourhood Statistics. Indices of Deprivation and Classification. 2011. http://www.neighbourhood.statistics.gov.uk (accessed 16 Jan 2012).

11 IMMQAS. Immunology Quality Services. http://www.immqas.org.uk

12 Novalite Monkey Cerebellum/Cerebrum \& Mouse Stomach Slides (Insert). May 2010. Revision 0 .

13 Glutamic Acid Decarboxylase (GAD) Autoantibody ELISA kit form RSR-Instructions for use (kit insert).

14 Novalite ICA Primate pancreas Slides (Insert). May 2010 Revision 0.

15 Klingensmith GJ, Pyle L, Arslanian S, et al; (TODAY Study Group). The Presence of GAD and IA-2 Antibodies in Youth with a Type 2 Diabetes Phenotype. Diabetes Care 2010;33:1970-5.

16 Awa WL, Boehm BO, Rosinger S, et al. HLA-typing, clinical, and immunological characterization of youth with type 2 diabetes mellitus phenotype from the German/ Austrian DPV database. Paediatr Diabetes 2013;14:562-74.

17 Savola K, Sabbah E, Kulmala P, et al. Autoantibodies associated with Type I diabetes mellitus persist after diagnosis in children. Diabetologia 1998:41:1293-7.

18 Brezar V, Carel JC, Boitard C, et al. Beyond the hormone: insulin as an autoimmune target in type 1 diabetes. Endocr Rev 2011;32:623-69.

19 Bingley PJ, Bonifacio E, Williams AJ, et al. Prediction of IDDM in the general population: strategies based on combinations of autoantibody markers. Diabetes 1997;46:1701-10.

20 Type 2 diabetes in children and adolescents. American diabetes association. Diabetes Care 2000;23:381-9.

21 Franck M, Paquot N, Scheen AJ. Influence of body weight on the natural history of and the therapeutic approaches to type 1 diabetes. Rev Med Liege 2012;67:461-7

22 Brophy S, Cooksey R, Gravenor MB, et al. Risk factors for childhood obesity at age 5: analysis of the millennium cohort study. BMC Public Health 2009; 9:467.

23 Fourlanos S, Dotta F, Greenbaum CJ, et al. Latent autoimmune diabetes in adults (LADA) should be less latent. Diabetologia 2005:48:2206-12.

24 Dabelea D, Bell RA, D'Agostino RB, et al. Incidence of diabetes in youth in the United States. JAMA 2007;297:2716-24.

25 Reinehr T, Schober E, Wiegand S, et al. Beta-cell autoantibodies in children with type 2 diabetes mellitus: subgroup or misclassification? Arch Dis Child 2006;91:473-7.

26 Van Deutekom AW, Heine RJ, Simsek S. The islet autoantibody titres: their clinical relevance in latent autoimmune diabetes in adults (LADA) and the classification of diabetes mellitus. Diabet Med 2008;25:117-25.

27 Tfayli H, Bacha F, Gungor N, et al. Phenotypic type 2 diabetes in obese youth: insulin sensitivity and secretion in islet cell antibody-negative versus -positive patients. Diabetes 2009;58:738-44.

28 Rodriguez BL, Fujimoto WY, Mayer-Davis EJ, et al. Prevalence of cardiovascular disease risk factors in U.S. children and adolescents with diabetes: the SEARCH for diabetes in youth study. Diabetes Care 2006;29:1891-6. 